\title{
The X-ray spectrum of the classical TTauri star TW Hydrae observed by LETGS aboard Chandra
}

\author{
A. J. J. Raassen ${ }^{1,2}$ \\ 1 SRON Netherlands Institute for Space Research, Sorbonnelaan 2, 3584 CA Utrecht, The Netherlands \\ 2 Astronomical Institute "Anton Pannekoek", Kruislaan 403, 1098 SJ Amsterdam, The Netherlands \\ e-mail: a.j.j.raassen@sron.nl
}

Received 18 November 2008 / Accepted 27 July 2009

\begin{abstract}
Aims. I present the analysis of the X-ray spectrum of the classical TTauri star TW Hydrae, and determine emission measure distributions, elemental abundances, electron temperatures, densities, and line fluxes.

Methods. TW Hya was observed in the wavelength range 5-175 A by CHANDRA-LETGS. I performed a multi-temperature fit, based on a collisional ionisation equilibrium (CIE) model for optically thin plasmas. I measured line fluxes by fitting a Gaussian line profile, folded through the response matrix, to the observed line features and I applied these values to establish plasma properties.

Results. No line features were established in the spectrum above $40 \AA$. Due to the absence of line features above $40 \AA N_{\mathrm{H}}$ is calculated to be somewhat higher than assumed so far. The observed "coronal" temperature covers a range from about 1.5 to $20 \mathrm{MK}$. The abundance pattern shows a very strong enhancement of $\mathrm{Ne}$ and some enhancement of N. From forbidden-intercombination line ratios of O VII and of Ne IX I obtain a value for the electron density of $n_{\mathrm{e}} \simeq 10^{12} \mathrm{~cm}^{-3}$. This is higher than the value for electron densities in coronal plasmas of older late type main-sequence stars. A blackbody component was added to describe the shape of the continuum around $15 \AA$ better. The emitted X-ray luminosity is in the order of $10^{30} \mathrm{erg} / \mathrm{s}$.
\end{abstract}

Key words. techniques: spectroscopic - stars: individual: TW Hya - stars: coronae

\section{Introduction}

TTauri stars (TTS) are young low-mass pre-main sequence stars, especially present in star-forming regions. They can be divided into two types: classical TTauri stars (cTTS) and weakline TTauri stars (wTTS). Different from the cTTS, the weakline TTauri stars do not show strong $\mathrm{H}_{\alpha}$ emission. Classical TTauri stars are surrounded by a disc of gas and dust, while the weak-line TTauri stars have no surrounding disc. Both types of TTauri stars are X-ray emitters. The X-rays were observed by the Einstein Satellite (e.g. Feigelson \& DeCampli 1981) and by ROSAT (e.g. Feigelson et al. 1993). The X-rays from weak-line TTauri stars are commonly interpreted as being produced by a scaled up coronal structure. The same is true for classical TTauri stars. However, in cTTS additional mechanisms might be present e.g., jets, winds, or accretion from the disc to the stellar surface. In the last case it is expected that the material funnels from the disc to the stellar surface along magnetic field lines, resulting in hot X-ray emitting spots. TW Hya belongs to the group of classical TTauri stars. It is spectral type K8V (SIMBAD) and nearby, at a distance of 56.4 parsec (Perryman et al. 1997a, b), recently corrected to 53.7 parsec (van Leeuwen 2007). It is surrounded by a circumstellar disc. A wide range of periods has been given over the years (e.g., Herbst \& Koret 1988; Mekkaden 1998; Rucinski et al. 2008). Recently, Setiawan et al. (2008) have concluded that a Jupiter-like planet is present close to the star, inside the disc and with a period of 3.56 days. However, Huélamo et al. (2008) state that a cool spot on the stellar surface is more likely to explain the observations.
Since the era of high-resolution X-ray spectroscopy started after the launches of Chandra and XMM-Newton, many papers on the high-resolution X-ray spectrum of TW Hya have appeared. The $R G S$ aboard XMM-Newton was used by Stelzer \& Schmitt (2004) and Robrade \& Schmitt (2006), while HETGS aboard Chandra was applied by Kastner et al. (2002) and Ness \& Schmitt (2005). Robrade \& Schmitt (2006) and Stelzer \& Schmitt (2004) made a fit to the spectrum with three temperature bins, while Stelzer \& Schmitt (2004), Kastner et al. (2002), and Ness \& Schmitt (2005) investigated individual lines and line fluxes. Güdel \& Telleschi (2007) studied the O VII/O VIII line ratio in an ensemble of wTTS and cTTS.

For RGS and HETGS the wavelength ranges are at the higher end limited to about $35 \AA$. Applying LETGS, however, I may extend the wavelength range to far higher wavelengths and check the effects of the models above the RGS and HETGS limits.

The strategy in this paper is to perform a multi-temperature fit to the total spectrum (Sect. 3). In this fitting procedure firstly two different models have been applied (CIE and NEI) and the optimal number of T-components has been determined. Results of the global fitting are compared with results from the analysis based on individual lines, described in Sect. 4. In Sect. 5 I discuss the agreements and differences in comparison with the papers mentioned above (Robrade \& Schmitt 2006; Stelzer \& Schmitt 2004; Kastner et al. 2002; and Ness \& Schmitt 2005).

\section{Observations}

The spectrum of TW Hya was obtained on August 22, 2006 during a $151.64 \mathrm{ks}$ observation using HRC-S in combination with 


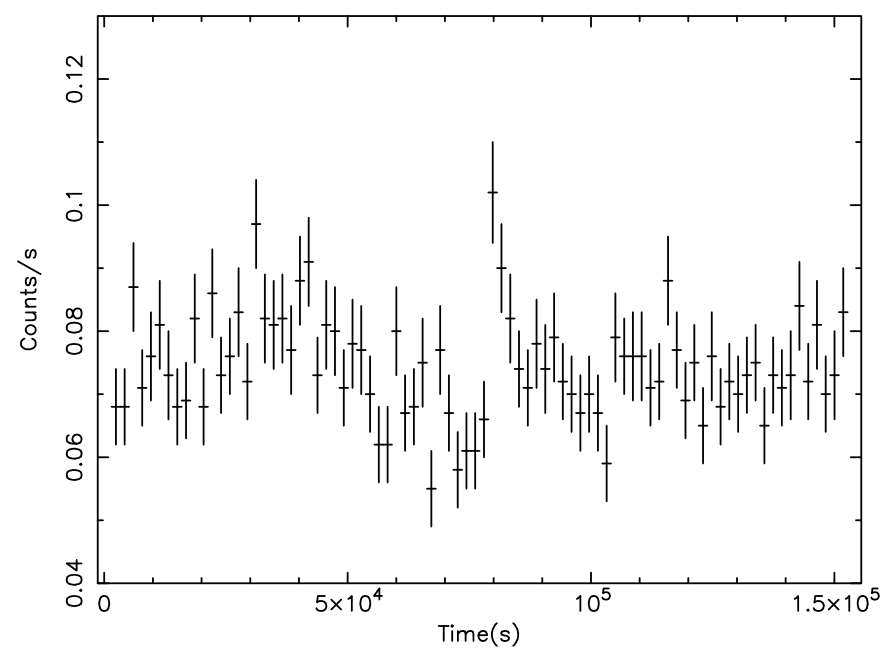

Fig. 1. The zeroth order lightcurve of the LETGS observation of TW Hya. The time bin size is $1800 \mathrm{~s}$.

Table 1. Observation log of the data of TW Hya.

\begin{tabular}{|c|c|c|c|}
\hline Obs ID Instrument & Grating & Start Date & Exp.(ks) \\
\hline $6443 \quad$ HRC-S & LETG & 2006-08-22T07:47:56 & 151.64 \\
\hline
\end{tabular}

LETG aboard CHANDRA. The log of the observations is shown in Table 1. The data were pipelined with CIAO3.4 with CALDB version 3.4.2 and prepared to be implemented in SPEX by the programs extract and crele developed at SRON.

In the lightcurve based on the total detector area some time bins showed saturation, i.e., they registered over 184 counts/s. Although this saturation will only sparely influence the spectral counts, the saturated time bins were excluded from the further procedure. Figure 1 shows the zeroth order lightcurve. The time bin size is $1800 \mathrm{~s}$. The average count rate is 0.07 counts/s. This lightcurve shows variability up to about $50 \%$ over the total exposure time. However, no specific time intervals could be selected to obtain a reliable spectrum of a "flare" state. No periodicity was established during the observation.

The observed LETGS spectrum covers the range from 1 to $176 \AA$. The final spectrum is the result of the sum of the spectrum of the +1 order and the -1 order. The background, which is high at longer wavelengths, was subtracted. No significant line features (apart from higher order lines) are established above $40 \AA$.

\section{Multi-temperature fitting}

\subsection{A 10-T CIE and NEI model as a starter}

I started the analysis of the LETGS-spectrum of TW Hya by means of two different spectral models: a model for optically thin plasma in collisional ionisation equilibrium (CIE) and a model for a non-equilibrium plasma (NEI). This approach was chosen to investigate the possibility of establishing different mechanisms for hot X-ray radiation i.e., a coronal part as for the wTTS and an accretion part from in-falling disc material. The two different models resulted in a solution with three significant temperature components only and with comparable $\chi^{2}$ values. However, the parameter $U\left(=n_{\mathrm{e}} \times t\right)$ in the NEI model obtained a value that high that equilibrium is reached and the NEI model mimics the CIE model.

From this point on I use a 3-T model for optically thin plasma in collisional ionisation equilibrium (CIE) as implemented in SPEX version 2.0 $0^{1}$ (Kaastra et al. 1996a) in combination with MEKAL (Mewe et al. 1995; Kaastra et al. 1996b). The ionisation equilibrium is based on calculations by Arnaud \& Rothenflug (1985).

\subsection{A 3-T model}

A 3-T model was fitted to the data over the total wavelength range from 5 to $175 \AA$. The T-parameters, emission measures and abundances were freely varied. Two temperature components, describing most of the line features, are established at $0.127 \mathrm{keV}$ and $0.264 \mathrm{keV}$. They describe very well the observed line structure above $10 \AA$. Based on the Mg XI, Mg XII and Si XIII, Si XIV line features below $10 \AA$ a third temperature component at $1.45 \mathrm{keV}$ is determined. A fourth T-component of $0.65 \mathrm{keV}$ appeared to model the continuum. These components are in agreement with the temperatures found by Robrade \& Schmitt (2006) and Stelzer \& Schmitt (2004) in a different way and based on observations with RGS on board of XMM-Newton.

However, fitting the spectrum with these four CIE components results in some deviations, i.e., the model continuum is a bit lower than the data around $15 \AA$ and a bit higher below $10 \AA$, while weak line features below $10 \AA$ are not recognised as lines by the model. I notice the same deviations when applying the parameter set given by Robrade \& Schmitt (2006). To solve this deficiency in the shape of the underlying continuum around $15 \AA$, a blackbody component was introduced, replacing the $T=0.65 \mathrm{keV}$ component. The blackbody component has a well determined temperature of $0.187 \mathrm{keV}$, just in between the two cool temperature components. This component is strongly related to the $0.60 \mathrm{keV}$ temperature component of Robrade \& Schmitt (2006), but doesn't affect the other T-components. From Table 2 of Günther et al. (2007) we notice that the CIE component at $0.7 \mathrm{keV}$ doesn't improve their fit very significantly. The blackbody component improved the shape of the continuum and it lowered the $\chi^{2}$ of the fit significantly. Due to the lower continuum below $10 \AA$ the weak line features appear more pronounced. It results in a higher value of the abundance of $\mathrm{Si}$, compared to the value given by Robrade \& Schmitt (2006). It is possible that my blackbody component takes into account many weak lines, that are not included in the models and produce a pseudocontinuum.

The spectrum and the model are shown in Fig. 2. The grey line (green in the electronic version) is the model fit to the spectrum.

The parameter values of the fit are collected in Table 2. This table shows the interstellar column density $N_{\mathrm{H}}$, the determined temperature values, the emission measures and the emitted luminosities of the different temperature bins, the electron densities, and the abundances. The emission measure is defined as $E M=n_{\mathrm{e}} n_{\mathrm{H}} V$, in which $N_{\mathrm{H}}=0.85 \times n_{\mathrm{e}}$. Except for the density, broadening, and $\mathrm{w}$-shift, the parameters were all fitted simultaneously.

The interstellar column density $N_{\mathrm{H}}$ was free to vary. Only one $N_{\mathrm{H}}$ parameter was used for all four components. However, it is not necessarily true that all plasma undergoes the same absorption. The continuum at higher wavelength is weak and

${ }^{1}$ http://www.sron.nl/ divisions/hea/spex/version2.0 


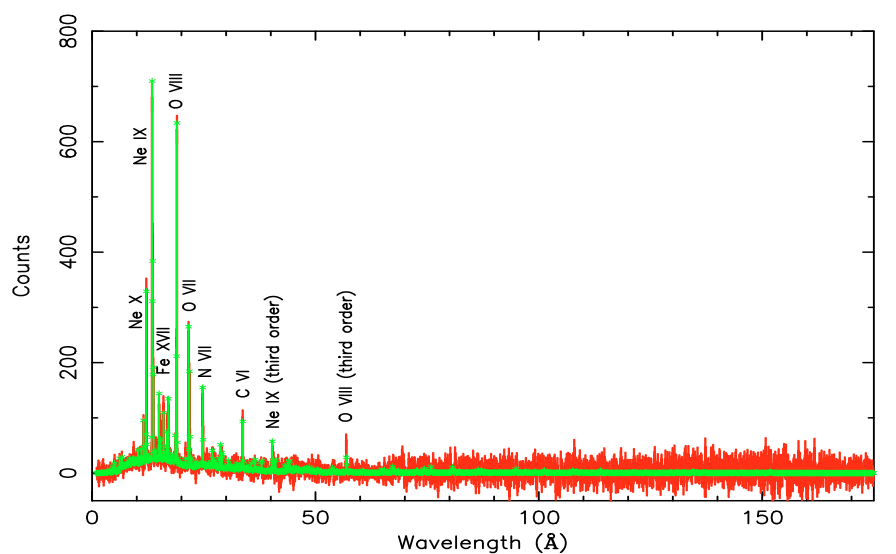

Table 2. Parameters of the 3-T fit to the spectrum of TW Hya.

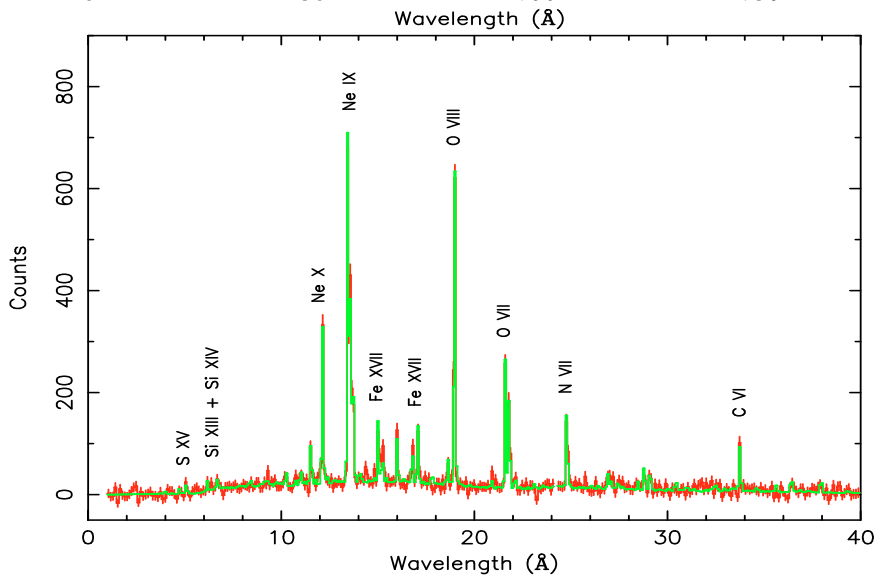

Fig. 2. The LETGS spectrum of TW Hya together with the fitted CIE model, grey line (green in the electronic version). The top panel shows the total spectrum and the bottom panel shows the area between 0 and 40 A.

not very reliable due to the high value of the subtracted background. However, line features or the absence of line features are indicative for the interstellar column density. The absence of the Ne VIII lines around 88 and $98 \AA$ indicates a value for $N_{\mathrm{H}} \geq 2 \times 10^{20} \mathrm{~cm}^{-2}$. My fitted value $\left(N_{\mathrm{H}}=5.2 \times 10^{20} \mathrm{~cm}^{-2}\right)$ is higher than that value and also slightly higher than the values used by Robrade \& Schmitt (2006) and Stelzer \& Schmitt (2004). This higher value is necessary to suppress Si XII line features near $44 \AA$ that appear in the model, but are absent in the observed spectrum. These lines are outside the wavelength range observed by Robrade \& Schmitt (2006) and Stelzer \& Schmitt (2004).

A slight shift in $\lambda(\approx 1.2 \mathrm{e}-4)$ was applied to optimise the positions of the model lines with respect to the data. This shift corresponds to about $35 \mathrm{~km} \mathrm{~s}^{-1}$. The value of $v_{\text {rad }}$ of TW Hya is $12.5 \mathrm{~km} \mathrm{~s}^{-1}$ (Reid 2003), while the satellite and earth orbital motion result in about $-12 \mathrm{~km} \mathrm{~s}^{-1}$ at time of observation. My fitted value is higher than the result of these geometrical motions. An instrumental wavelength calibration bias is likely on forehand and cannot be ruled out.

A line broadening parameter, which corresponds to a velocity broadening of $287 \mathrm{~km} \mathrm{~s}^{-1}$, was added to improve the fit. This parameter is added to the instrumental line spread function, which is taken into account by means of folding the model with the response matrix. Ness \& Schmitt (2005) obtain the best fit results by introducing an FWHM of $0.016 \AA$ for all lines of their HETGS observation. This is equivalent to about $200 \mathrm{~km} \mathrm{~s}^{-1}$.

\begin{tabular}{|c|c|c|}
\hline Parameters & & \\
\hline $\begin{array}{l}\log N_{\mathrm{H}}\left[\mathrm{cm}^{-2}\right] \\
\text { distance } \\
\text { w-shift } \\
\text { broadening } \\
T_{1}[\mathrm{keV}] \\
T_{2}[\mathrm{keV}] \\
T_{3}[\mathrm{keV}] \\
T_{\mathrm{Bb}}[\mathrm{keV}] \\
\end{array}$ & $\begin{array}{c}20.72(.09) \\
53.7 \mathrm{pc} \\
1.2 \mathrm{e}-4 \\
287 \mathrm{~km} \mathrm{~s}^{-1} \\
0.127(.012) \\
0.264(.007) \\
1.45(.43) \\
0.187(.016)\end{array}$ & \\
\hline $\begin{array}{l}E M_{1}\left[10^{51} \mathrm{~cm}^{-3}\right] \\
E M_{2}\left[10^{51} \mathrm{~cm}^{-3}\right] \\
E M_{3}\left[10^{51} \mathrm{~cm}^{-3}\right] \\
\text { Area }_{\mathrm{Bb}}\left[10^{8} \mathrm{~cm}^{2}\right]\end{array}$ & $\begin{array}{c}11.5(3.3) \\
26.3(2.1) \\
9.3(2.5) \\
3.57(1.7) \\
\end{array}$ & \\
\hline $\begin{array}{l}n_{\mathrm{e}} \mathrm{O} \text { VII }\left[10^{11} \mathrm{~cm}^{-3}\right] \\
n_{\mathrm{e}} \mathrm{Ne} \operatorname{IX}\left[10^{12} \mathrm{~cm}^{-3}\right]\end{array}$ & $\begin{array}{l}6.3(2.6) \\
2.5(0.4) \\
\end{array}$ & \\
\hline $\begin{array}{l}L_{\mathrm{X} 1}\left[10^{30} \mathrm{erg} / \mathrm{s}\right]^{a} \\
L_{\mathrm{X} 2}\left[10^{30} \mathrm{erg} / \mathrm{s}\right] \\
L_{\mathrm{X} 3}\left[10^{30} \mathrm{erg} / \mathrm{s}\right] \\
L_{\mathrm{Bb}}\left[10^{30} \mathrm{erg} / \mathrm{s}\right] \\
L_{\mathrm{Tot}}\left[10^{30} \mathrm{erg} / \mathrm{s}\right]\end{array}$ & $\begin{array}{l}0.14(.04) \\
0.83(.07) \\
0.29(.08) \\
0.43(.20) \\
1.69(.22) \\
\end{array}$ & \\
\hline $\begin{array}{l}\mathrm{C} \\
\mathrm{N} \\
\mathrm{O} \\
\mathrm{Ne} \\
\mathrm{Mg} \\
\mathrm{Si} \\
\mathrm{Fe} \\
\mathrm{Ni}\end{array}$ & $\begin{array}{l}1.26(.23) \\
2.87(.31) \\
1.00(.04) \\
10.3(.34) \\
1.61(.89) \\
1.35(.43) \\
0.61(.06) \\
0.70(.49)\end{array}$ & $\begin{array}{c}A / \mathrm{O} \\
1.26(.24) \\
2.87(.33) \\
1.00(.06) \\
10.3(.53) \\
1.61(.89) \\
1.35(.43) \\
0.61(.06) \\
0.70(.49)\end{array}$ \\
\hline $\begin{array}{l}\chi^{2} / \text { d.o.f. } \\
\chi_{\text {red }}^{2}\end{array}$ & $\begin{array}{c}1564 / 1536 \\
1.02 \\
\end{array}$ & \\
\hline
\end{tabular}

Notes: Statistical $1 \sigma$ errors are in parentheses. The emitted luminosity $\left(L_{\mathrm{X}}\right)$ is given in the range from 0.3 to $10 \mathrm{keV}$.

They do not mention whether this broadening is included with the instrumental broadening or not.

The densities given in Table 2 are obtained by fitting to the line triplets of He-like ions (see also Sect. 4.3) in a narrow selected wavelength range around $22 \AA$ (O VII) and around $13.5 \AA$ (Ne IX) only. During this fit procedure the oxygen and neon abundances and electron density $\left(n_{\mathrm{e}}\right)$ were free to vary, while all other parameters were fixed at values obtained in the overall fitting procedure. The abundance served as normalisation parameter. The obtained electron densities are $n_{\mathrm{e}}=6.3(2.6) \times 10^{11} \mathrm{~cm}^{-3}$ based on the O VII line triplet and $n_{\mathrm{e}}=2.5(0.4) \times 10^{12} \mathrm{~cm}^{-3}$ based on Ne IX.

The emitted luminosities are calculated over the energy range from 0.3 to $10 \mathrm{keV}$. They are obtained for all given temperature bins. The uncertainty in the luminosities is assumed to be the same as the relative uncertainties of the emission measures. Robrade \& Schmitt (2006) and Stelzer \& Schmitt (2004) give luminosity values in slightly different energy ranges. In their energy ranges, $0.2-10 \mathrm{keV}$ and $0.45-2.25 \mathrm{keV}$, respectively, they obtain $2.0 \times 10^{30} \mathrm{erg} / \mathrm{s}$ and $1.4 \times 10^{30} \mathrm{erg} / \mathrm{s}$. The luminosities in these ranges obtained in the model fit of this study (corrected for the distance they use) are $2.05 \times 10^{30} \mathrm{erg} / \mathrm{s}$ and $1.47 \times 10^{30} \mathrm{erg} / \mathrm{s}$, respectively. They are in very good agreement with those of Robrade \& Schmitt (2006) and Stelzer \& Schmitt (2004). It shows that the luminosity is a very robust quantity. About $55 \%$ of the emitted luminosity is due to line radiation, 
while the resulting $45 \%$ is radiated in the continuum or a pseudocontinuum produced by many weak indistinguishable lines not present in the models. These percentages are comparable with values $(60 \%$ versus $40 \%)$ obtained using the parameter set of Robrade \& Schmitt (2004).

The abundances are coupled for the three temperature components. This is done to stabilise the fit procedure and is not based on physical grounds. The abundances are relative to solar photospheric values (Anders \& Grevesse 1989), except for iron. For Fe the value of Grevesse \& Sauval (1999) has been applied. It differs by a factor 1.45 . For the obtained abundances the over-abundance of neon (a factor of ten higher than oxygen) is most remarkable, while the abundance of nitrogen is a factor 3 higher in comparison with oxygen. The other abundances are close to solar. Iron seems to be depleted. My absolute abundance values are far higher than values given by Stelzer \& Schmitt (2004). This can be explained by the fact that the abundances are strongly anti-correlated with the emission measures. Therefore in many papers an abundance ratio related to the abundance of an arbitrarily chosen, generally dominantly present element is given. The abundance pattern, i.e., abundances relative to oxygen $(A / O)$, agrees very well with the results of Stelzer \& Schmitt (2004). My carbon abundance is somewhat higher as a result of my higher $N_{\mathrm{H}}$ value. The quantity $A \times E M$ is robust. My values for the emission measures are far lower than those in the paper by Stelzer \& Schmitt (2004). However, the ratio between the $E M_{2}$ and $E M_{1}$, given by them as 2.35 , is the same as in my fit i.e., 2.36 .

The errors given in the paper are quite different for different quantities. The $\mathrm{T}$ parameters, emitted luminosities, abundance ratios $(A / \mathrm{O})$, the observed line fluxes, and $A \times E M$, given in Sect. 4 are robust. Their statistical $1 \sigma$ errors given in Tables $2-4$ are robust. For the abundances and the emission measures, however, the errors given in Table 2 are statistical errors that belong to the given fit-parameters. The absolute values of the abundance and emission measures might change considerably more as long as the abundance ratios $(A / \mathrm{O})$ and $A \times E M$ remain unchanged.

\section{Individual line fluxes}

The individual observed line photon fluxes of the LETGS spectrum of TW Hya have been measured. Many line features are located between 10 and $25 \AA$. Unfortunately, the number of line features above $40 \AA$ are scarce. The most obvious features above $40 \AA$ are higher order lines of oxygen and neon. Lines below $10 \AA$ are weak, but definitely present, as they are well positioned in relation to experimental laboratory wavelengths in databases (Kelly 1987, and NIST ${ }^{2}$ ).

For each observed line a Gaussian profile was folded through the response matrix and fitted to the line feature together with a power-law taking into account for the continuum. For lines used for the temperature and density determination in Sect. 4.3 also the following (maybe more sophisticated) method was applied. This was done to avoid contributions by blends. After the final overall multi-temperature fit (Sect. 3.2), the three wavelength ranges that belong to He-like ions (28.5-29.8 $\AA$ for N VI), (21.4-22.4 $\AA$ for O VII, and 13.2-14 $\AA$ for Ne IX) were selected. Then the ions N VI, O VII, and Ne IX were ignored from the model. Now the model describes the total spectrum except the He-like line triplet of the ignored ions. These lines were measured by folding a Gaussian through the response matrix and by

2 http://physics.nist.gov/PhysRefData/ASD/index.html
Table 3. The observed line photon fluxes.

\begin{tabular}{|c|c|c|c|c|}
\hline ion & $\begin{array}{l}\lambda_{0} \\
{[\AA]}\end{array}$ & $\begin{array}{l}\lambda_{\text {obs }} \\
{[\AA]}\end{array}$ & $\begin{array}{c}F W H M \\
{[\AA]}\end{array}$ & Observed flux \\
\hline $\mathrm{S} \times \mathrm{V}$ & 5.064 & $5.061(.035)$ & - & $16.6(11.8)$ \\
\hline Si XIV & 6.182 & $6.200(.018)$ & - & $3.9(2.3)$ \\
\hline Si XIII & 6.648 & $6.656(.023)$ & - & $3.4(2.2)$ \\
\hline Si XIII & 6.688 & $6.698(.041)$ & - & $2.4(2.2)$ \\
\hline Si XIII & 6.740 & $6.766(.027)$ & - & $2.7(2.2)$ \\
\hline Mg XII & 8.423 & $8.474(-)$ & - & $4.2(-)$ \\
\hline Mg XI & 9.169 & $9.168(.014)$ & - & $6.4(3.4)$ \\
\hline Mg XI & 9.315 & $9.318(.018)$ & - & $12.0(6.2)$ \\
\hline $\mathrm{NeX}$ & 9.708 & $9.689(.032)$ & - & $7.6(4.8)$ \\
\hline $\operatorname{NeX}$ & 10.239 & $10.225(.011)$ & - & $11.5(4.5)$ \\
\hline Ne IX & 10.765 & $10.759(.012)$ & - & 7.7( 3.7$)$ \\
\hline $\mathrm{Ne}$ IX & 11.001 & 10.994(.008) & 一 & $12.6(4.2)$ \\
\hline Ne IX & 11.544 & 11.543(.006) & - & $22.0(4.8)$ \\
\hline $\mathrm{NeX}$ & 12.134 & $12.135(.003)$ & $0.017(.014)$ & $89.6(7.8)$ \\
\hline $\mathrm{Ne} I \mathrm{X}$ & 13.447 & $13.452(.002)$ & $0.024(.007)$ & $215.0(11.0)$ \\
\hline $\mathrm{Ne} I X$ & 13.553 & $13.555(.002)$ & $\lesssim 0.020$ & $129.0(9.0)$ \\
\hline Ne IX & 13.700 & $13.699(.003)$ & $0.041(.011)$ & $81.2(7.7)$ \\
\hline Ni XIX & 14.040 & $14.058(.028)$ & $\lesssim 0.093$ & $5.6(4.4)$ \\
\hline Ni XVIII & 14.081 & & & \\
\hline Ni XVIII & 14.370 & $14.381(.033)$ & $\lesssim 0.120$ & $10.2(6.6)$ \\
\hline O VIII & 14.821 & $14.824(.014)$ & - & $6.9(2.8)$ \\
\hline Fe XVII & 15.013 & 15.031(.008) & $0.070(.022)$ & $35.9(6.5)$ \\
\hline O VIII & 15.176 & $15.174(.020)$ & fix & $9.0(4.1)$ \\
\hline Fe XVII & 15.260 & $15.258(.008)$ & $\lesssim 0.056$ & $17.4(5.1)$ \\
\hline O VIII & 16.006 & 16.010(.006) & $\lesssim 0.053$ & $29.7(5.4)$ \\
\hline Fe XVII & 16.775 & $16.790(.006)$ & $\lesssim 0.041$ & $20.4(4.6)$ \\
\hline Fe XVII & 17.051 & $17.056(.060)$ & fix at 0 & $22.9(12.6)$ \\
\hline Fe XVII & 17.100 & $17.098(.039)$ & fix at 0 & $15.1(10.3)$ \\
\hline O VII & 18.627 & 18.634(.018) & $0.066(.060)$ & $17.5(6.7)$ \\
\hline O VIII & 18.969 & 18.972(.002) & $0.027(.006)$ & $229.0(12.0)$ \\
\hline N VII & 20.910 & 20.917(.019) & 0.069 & 20.7( 8.6) \\
\hline O VII & 21.602 & $21.610(.003)$ & $\lesssim 0.043$ & $119.0(12.0)$ \\
\hline O VII & 21.804 & $21.807(.003)$ & $0.030(.011)$ & $103.0(11.0)$ \\
\hline O VII & 22.101 & $22.105(.015)$ & 0.035 & $15.3(6.3)$ \\
\hline N VII & 24.781 & 24.794(.005) & $0.046(.018)$ & 81.2(11.3) \\
\hline N VI & 28.787 & 28.799(fix) & $0.138(.103)$ & $31.2(12.0)$ \\
\hline N VI & 29.084 & 29.096(fix) & $\lesssim 0.146$ & $27.1(10.0)$ \\
\hline N VI & 29.536 & 29.548(fix) & - & $6.0(6.3)$ \\
\hline C VI & 33.736 & $33.743(.005)$ & - & $59.3(11.0)$ \\
\hline
\end{tabular}

Notes: Statistical $1 \sigma$ errors are in parentheses. $\lambda_{0}$ is the theoretical wavelength from Kelly (1987). $\lambda_{\mathrm{obs}}$ is the observed wavelength. The flux is in units $10^{-6}$ photons $\mathrm{cm}^{-2} \mathrm{~s}^{-1}$.

fitting to the lines, as described in Sect. 4, but without the additional power-law, since the continuum is taken into account by the multi-temperature model. During this fit all parameters of the model were fixed, except the Gaussians. This way the positions, observed photon fluxes, and widths $(F W H M)$ of the lines were established. Thanks to the folding of the Gaussian profile through the response matrix and the Line-Spread-Function (LSF) the given FWHM are net values, in addition to the instrumental width. The measured wavelengths, widths and line photon fluxes are collected in Table 3 together with the theoretical wavelengths from laboratory measurements (Kelly 1987).

Especially at higher wavelengths the lines are slightly shifted to longer wavelength. Unfortunately the line features do not extent to wavelengths above $40 \AA$. An underlying instrumental wavelength imperfection, cannot be ruled out. However, all lines given by Stelzer \& Schmitt (2004) in their Table 2, based on observations with $R G S$, are (even more pronounced) shifted to longer wavelengths, albeit they do not report this shift and 
they do not give error estimates for their wavelength measurements. Moreover, Günther \& Schmitt (2008) measure positive line shifts of the same order of magnitude, based on observations with FUSE and HST (see their Tables 4 and 5). It should be kept in mind that these observations concern another wavelength area and lower stages of ionisation. Therefore they might be related to other physical processes. The observed wavelength shift in the X-ray regime seems to be real. If not from physical origin, the observed shift would imply a calibration issue for both instruments (LETGS and RGS).

For a number of lines an FWHM could be obtained. However, the line widths are in many cases upper limits. The $F W H M$ of a line can be linked to a velocity ( $v$ ) by the formula: $F W H M(\AA) / \lambda_{\text {obs }}=2 \sqrt{\ln 2} v / c$. Applying this formula to the stronger isolated lines at $13.447 \AA, 18.969 \AA, 21.602 \AA$, and $24.781 \AA$, I obtain values for $v$ of 321(94), 256(57), $\lesssim 358$, and $334(130) \mathrm{km} \mathrm{s}^{-1}$, respectively. In Sect. $3.2 \mathrm{I}$ have obtained an overall broadening parameter $\left(v=287 \mathrm{~km} \mathrm{~s}^{-1}\right)$ to improve the multi-temperature fit; a value comparable with these values. $F W H M$ 's of the order of a few hundreds of $\mathrm{km} \mathrm{s}^{-1}$ are also found by Günther \& Schmitt based on FUSE observations, but in a wavelength area around $1000 \AA$ and based on cooler ions. Their broadening might originate from other processes and not directly be linked to the broadening in the X-ray spectrum.

The last column shows the observed line photon fluxes as measured at Earth in units $10^{-6}$ photons $\mathrm{cm}^{-2} \mathrm{~s}^{-1}$. The lines of $\mathrm{H}$-like neon, oxygen, nitrogen, and carbon are strong, as well as He-like lines of neon and oxygen. For these lines the uncertainties of the flux values are of the order of $10 \%$. The iron lines are moderately strong. Especially for neon and oxygen higher Rydberg states are visible up to $n=5$. Their relative uncertainties are higher.

\subsection{Emission measures from individual line fluxes}

Line fluxes $(F)$, emission measure $(E M)$, and abundances $(A)$ are related to each other by means of the formula:

$F=\left(4.1807 \times 10^{-54} \times \lambda \times A \times E M \times 10^{-s}\right) / \mathrm{d} p^{2}$.

In this formula $F$ is the line flux in units of photons $\mathrm{cm}^{-2} \mathrm{~s}^{-1}, \lambda$ is the wavelength of the line in $\AA, A$ is the abundance relative to solar photospheric values, EM is the emission measure in units $\mathrm{cm}^{-3}, \mathrm{~d} p$ is the distance of the source in parsecs and $s$ is the emissivity factor given by Mewe et al. (1985), applied for the temperature $\left(T_{\mathrm{m}}\right)$ of maximum emissivity. In Table 4 the values of the quantity $A \times E M$ obtained from individual line fluxes are given. The given uncertainties in this quantity are based on the uncertainties of the measured line fluxes and an assumed average uncertainty of $15 \%$ in the theoretical transition probabilities of the lines. The latter value might be a bit low for weak lines and a bit too high for strong resonance transitions. Since the forbidden and intercombination lines in He-like ions behave as communicating tubes under influence of the density I have used the sum $(\mathrm{f}+\mathrm{i})$ in this table.

From the table we notice that the value of $A \times E M$ differ up to a factor of 10 . For comparable values of $T_{\mathrm{m}}$ the values of $E M$, however, should be the same. The difference in value of $A \times E M$ in these cases is caused by deviations from solar abundance values for the involved ions. As an example: the ions O VII and $\mathrm{N}$ VII, although appearing in the same temperature range, show a difference of a factor 3 in the values of $A \times E M$. This indicates an overabundance of nitrogen compared to oxygen by a factor of
Table 4. Calculated values of $A \times E M$, based on individual line fluxes.

\begin{tabular}{lcrccr}
\hline \hline Ion & $\begin{array}{c}\lambda_{0} \\
{[\AA]}\end{array}$ & Flux & $\log T_{\mathrm{m}}$ & $s$ & $\begin{array}{c}A \times E M \\
\mathrm{~cm}^{-3}\end{array}$ \\
\hline Si XIV & 6.182 & $3.9(2.3)$ & 7.22 & 1.58 & $1.66(1.01)$ \\
Si XIII & 6.648 & $3.4(2.2)$ & 7.00 & 1.64 & $1.54(1.02)$ \\
Si XIII & $\mathrm{f}+\mathrm{i}$ & $5.1(3.1)$ & 7.00 & 1.77 & $3.09(1.93)$ \\
Mg XII & 8.423 & $4.2\left(-\frac{-)}{7.00}\right.$ & 1.86 & $2.49(--)$ \\
Mg XI & 9.169 & $6.4(3.4)$ & 6.81 & 1.70 & $2.41(1.33)$ \\
Mg XI & 9.314 & $12.0(6.2)$ & 6.81 & 1.79 & $5.47(2.94)$ \\
Ne X & 9.708 & $7.6(4.8)$ & 6.79 & 2.70 & $27.1(17.6)$ \\
Ne X & 10.239 & $11.5(4.5)$ & 6.78 & 2.10 & $9.79(4.10)$ \\
Ne IX & 10.765 & $7.7(3.7)$ & 6.60 & 2.95 & $44.00(22.1)$ \\
Ne IX & 11.001 & $12.6(4.2)$ & 6.60 & 2.61 & $32.20(11.8)$ \\
Ne IX & 11.544 & $22.0(4.8)$ & 6.59 & 2.14 & $18.10(4.79)$ \\
Ne X & 12.134 & $89.6(7.8)$ & 6.77 & 1.18 & $7.71(1.34)$ \\
Ne IX & 13.447 & $215.0(11.0)$ & 6.59 & 1.31 & $22.50(3.57)$ \\
Ne IX & $\mathrm{f}+\mathrm{i}$ & $210.2(11.8)$ & 6.59 & 1.36 & $24.40(3.90)$ \\
Fe XVII & 15.013 & $35.9(6.5)$ & 6.72 & 0.66 & $0.75(0.18)$ \\
O VIII & 15.176 & $9.0(4.1)$ & 6.51 & 2.09 & $5.03(2.41)$ \\
Fe XVII & 15.260 & $17.4(5.1)$ & 6.72 & 1.21 & $1.28(0.42)$ \\
O VIII & 16.006 & $29.7(5.4)$ & 6.50 & 1.48 & $3.86(0.91)$ \\
Fe XVII & 16.775 & $20.4(4.6)$ & 6.71 & 0.98 & $0.80(0.22)$ \\
Fe XVII & $17.05+.10$ & $38.0(16.3)$ & 6.71 & 0.67 & $0.72(0.33)$ \\
O VII & 18.627 & $17.5(6.7)$ & 6.34 & 1.51 & $2.09(0.86)$ \\
O VIII & 18.969 & $229.0(12.0)$ & 6.48 & 0.52 & $2.76(0.44)$ \\
N VII & 20.910 & $20.7(8.6)$ & 6.34 & 2.47 & $20.10(8.88)$ \\
O VII & 21.602 & $119.0(12.0)$ & 6.33 & 0.64 & $1.66(0.30)$ \\
O VII & $\mathrm{f}+\mathrm{i}$ & $118.3(12.7)$ & 6.32 & 0.69 & $1.81(0.33)$ \\
N VII & 24.781 & $81.2(11.3)$ & 6.32 & 1.48 & $6.83(1.40)$ \\
N VI & 28.787 & $31.2(12.0)$ & 6.17 & 1.67 & $3.50(1.44)$ \\
N VI & $\mathrm{f}+\mathrm{i}$ & $33.1(11.8)$ & 6.16 & 1.70 & $3.93(1.52)$ \\
C VI & 33.736 & $59.3(11.0)$ & 6.13 & 0.94 & $1.06(0.25)$ \\
\hline
\end{tabular}

Notes: $A$ is the elemental abundance and $E M$ the emission measure. $T_{\mathrm{m}}$ and $s$ are the temperature of maximum emissivity of the line and the corresponding line emissivity factor. $\lambda_{0}$ is the theoretical wavelength from Kelly (1987). Fluxes are given in units $10^{-6}$ photons $\mathrm{cm}^{-2} \mathrm{~s}^{-1} . A \times E M$ is given in $10^{52} \mathrm{~cm}^{-3}$. The error in $A \times E M$ includes the relative error in the flux values and a $15 \%$ error in the values for transition probabilities, imbedded in "s".

about 3. The same way (based on O VIII and Ne IX), an overabundance of neon compared to oxygen is established by a factor of about 10. Absolute values of the abundances are difficult to determine on the basis of line fluxes, since they are anti-correlated with the emission measure. $A \times E M$ is a robust quantity.

\subsection{Comparison with observed line photon fluxes from other papers}

In this subsection I compare the observed line photon fluxes obtained in this work with the most recent results from highresolution X-ray spectroscopy, i.e., Stelzer \& Schmitt (2004) using RGS aboard XMM-Newton, Kastner et al. (2002), and Ness \& Schmitt (2005), applying HETGS aboard Chandra (see Table 5). The values given by Ness \& Schmitt (2005) have been recalculated from energy fluxes to photon fluxes. For quite a number of lines the comparison is satisfactory, taking into account the uncertainties in the obtained fluxes. However, some severe deviations that exceed the given uncertainties are noticed. This is especially true for the comparison between the work of Stelzer \& Schmitt (2004) and Kastner et al. (2002).

The deviations are not limited to weak lines with high uncertainties in their measured flux values, but they appear also for the strong Ne IX lines around $13.5 \AA$ and the strong O VIII line 
Table 5. Comparison of observed line photon fluxes.

\begin{tabular}{|c|c|c|c|c|c|}
\hline ion & $\begin{array}{l}\lambda_{0} \\
{[\AA]}\end{array}$ & $\begin{array}{r}\text { Flux } \\
\text { this work }\end{array}$ & $\begin{array}{r}\text { Flux } \\
\text { Stelzer }\end{array}$ & $\begin{array}{r}\text { Flux } \\
\text { Kastner }\end{array}$ & $\begin{array}{l}\text { Flux } \\
\text { Ness }\end{array}$ \\
\hline Si XIV & 6.182 & $3.9(2.3)$ & - & $2.7(1.9)$ & - \\
\hline II & 6.6 & $4(2.2)$ & - & $2.1(1.7)$ & - \\
\hline & & 2.2) & - & 1.2) & - \\
\hline & & $7(2.2)$ & - & $1.4(1$. & - \\
\hline XIII & $f+i$ & $5.1(3.1)$ & - & $2.2(1.8)$ & - \\
\hline g XII & 8.4 & $4.2(-)$ & - & $3.0(2.0)$ & - \\
\hline$x$ & 9.7 & $7.6(4.8)$ & - & $2.0(2.1)$ & \\
\hline ex & 10.239 & $11.5(4.5)$ & 一 & $7.8(2.8)$ & $1.2(1.8)$ \\
\hline eIX & 544 & $22.0(4.8)$ & - & $18.0(4.6)$ & \\
\hline$e x$ & .134 & $89.6(7.8)$ & $63(15)$ & $73.7(7.8)$ & 7.0) \\
\hline e IX & 3.447 & $215.0(11.0)$ & $231(22)$ & $122.4(11.9)$ & - \\
\hline eIX & 13 & $129.0(9.0)$ & 174(11) & $79.2(10.5)$ & - \\
\hline IX & 13.700 & $81.2(7.7)$ & $82(15)$ & $35.3(8.6)$ & - \\
\hline Ne IX & $f+i$ & $210.2(11.8)$ & 256(19) & $114.5(13.6)$ & \\
\hline Fe XVI & 15.013 & $35.9(6.5)$ & - & $\lesssim 179.0$ & $46.2(8.1)$ \\
\hline O VIII & 15.176 & $9.0(4.1)$ & - & $\lesssim 15.9$ & - \\
\hline $\mathrm{F}$ & 260 & $17.4(5.1)$ & - & $23.2(8.4)$ & 5.4) \\
\hline & 006 & $29.7(5.4)$ & $35(11)$ & $35.7(10.4)$ & 7.8) \\
\hline $\mathrm{F}$ & 16.775 & $20.4(4.6)$ & 一 & $15.3(9.9)$ & 5.7) \\
\hline Fex & 17.051 & $22.9(12.6)$ & $27(9)$ & $35.9(13.1)$ & 9.0) \\
\hline Fe XVI & 17.100 & $15.1(10.3)$ & - & $25.4(12.7)$ & 6.9) \\
\hline O VII & 3.627 & $17.5(6.7)$ & $17(4)$ & $10.8(14.1)$ & \\
\hline O VIII & 18.9 & $0(12.0)$ & $304(28)$ & $7(28.0)$ & 26.5) \\
\hline & 21. & & $174(21)$ & $91.3(36.3)$ & - \\
\hline & & & $95(15)$ & $105.4(40.4)$ & - \\
\hline O VII & 22.101 & $15.3(6.3)$ & 4(6) & $\lesssim 57.0$ & - \\
\hline O VII & $\mathrm{f}+\mathrm{i}$ & $118.3(12.7)$ & $99(16)$ & $\lesssim 162$ & - \\
\hline $\mathrm{N}$ & 2 & & & $87.8(36.5)$ & - \\
\hline N VI & 28.7 & $.2(12.0)$ & $29(13)$ & - & - \\
\hline N VI & 29.084 & $.1(10.0)$ & $13(6)$ & - & - \\
\hline N VI & 29.536 & $0(6.0)$ & $4(5)$ & 一 & - \\
\hline N VI & $\mathrm{f}+\mathrm{i}$ & 3.1(11.7) & $17(8)$ & 一 & - \\
\hline C VI & 33.736 & $59.3(11.0)$ & $78(14)$ & - & - \\
\hline
\end{tabular}

Notes: This work, Stelzer \& Schmitt (2004), Kastner et al. (2002), and Ness \& Schmitt (2005). $\lambda_{0}$ is the theoretical wavelength from Kelly (1987). Flux in units $10^{-6}$ photons $\mathrm{cm}^{-2} \mathrm{~s}^{-1}$.

at $19.0 \AA$ (see Fig. 3). These figures show the observed line photon fluxes measured by Stelzer \& Schmitt (2004), Kastner et al. (2002), and this work versus wavelength and versus temperature of maximum emissivity of the line. From these figures it is difficult to conclude whether the differences are wavelength dependent, and therefore due to the use of three different instruments, or temperature dependent, and therefore related to variability of emission measures in the source TW Hya. The line ratios of the Ne IX lines, however, are comparable for the three spectra (see also the next section).

\subsection{Temperature and density determination based on individual line flux ratios}

The ratios between the line fluxes of the resonance lines from two ionisation stages of the same elements provide valuable information on the temperature regime of the plasma. Apart from that the line triplets in the He-like ions, consisting of a resonance line (r), an intercombination line (i) and a forbidden line (f), are strong diagnostic tools for temperature as well as for density (Gabriel \& Jordan 1969).
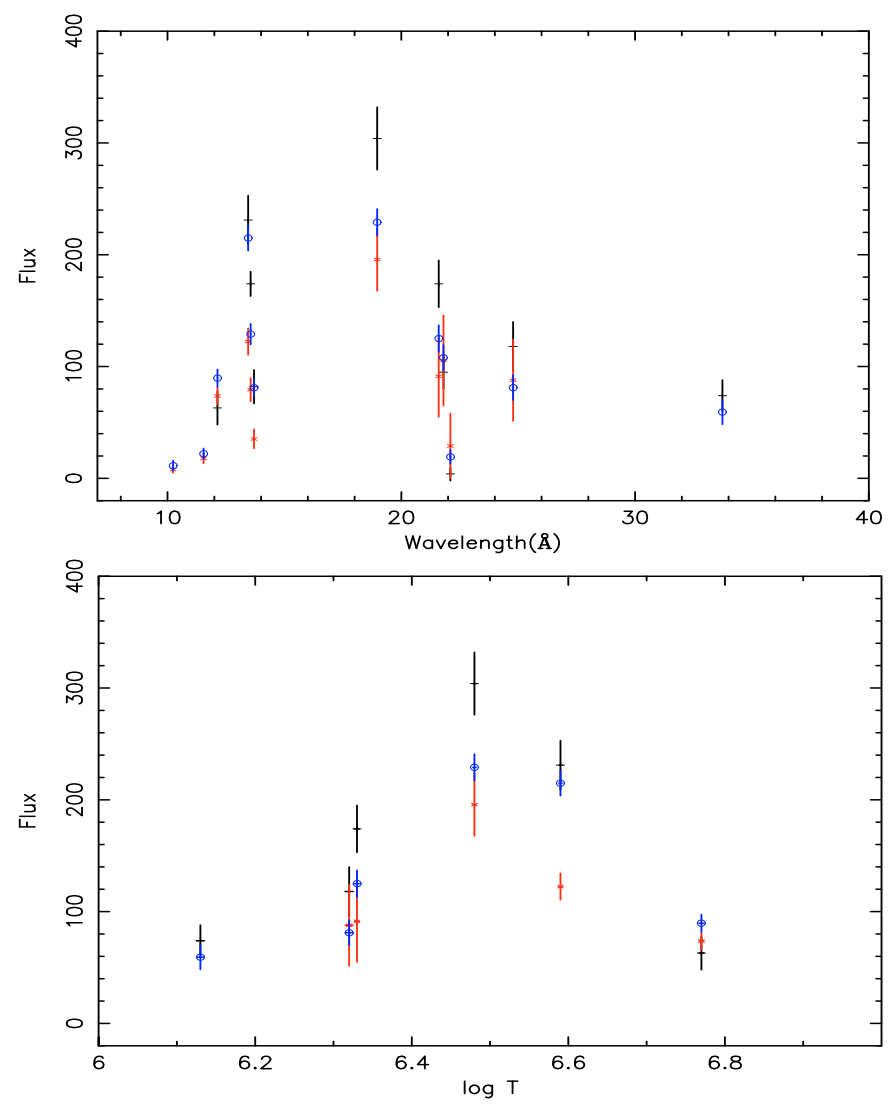

Fig. 3. Line fluxes versus wavelength (top) and versus $\log T$ (bottom) from: - Stelzer \& Schmitt (2004), * Kastner et al. (2002) and ${ }^{\circ}$ This work (black, red, and blue in the electronic version, respectively). Fluxes are given in $10^{-6}$ photons $\mathrm{cm}^{-2} \mathrm{~s}^{-1}$.

\subsubsection{Temperature}

The temperature can be established, based on individual line fluxes. The ratio between the summed fluxes of the line triplets in the He-like ions and the resonance lines of $\mathrm{H}$-like ions as well as the ratio $(\mathrm{f}+\mathrm{i}) / \mathrm{r}$ in the He-like line-triplets are temperature sensitive. As values for the ratios between the He-like line triplets and $\mathrm{H}$-like resonance lines I obtain 0.79(.23) for N , 1.04(.09) for $\mathrm{O}, 4.74(.45)$ for $\mathrm{Ne}$, and 2.2(1.6) for $\mathrm{Si}$, resulting in temperatures of $0.180(.022) \mathrm{keV}, 0.244(.007) \mathrm{keV}, 0.296(.007) \mathrm{keV}$, and $1.13(0.50) \mathrm{keV}$, respectively. These results are based on tables of line-emissivities given by Mewe et al. (1985) and MEKAL. My ratio for $\mathrm{O}$, as well as my $N_{\mathrm{H}}$ value are a bit higher than the values used by Güdel \& Telleschi (2007) in their Fig. 2.

As ratios $(\mathrm{f}+\mathrm{i}) / \mathrm{r}$ in the He-like ions $\mathrm{N}$ VI, O VII, and Ne IX the values of $1.06(.55), 0.994(.146)$, and $0.978(.074)$ were established, respectively. These values correspond to temperatures of $\lesssim 0.28 \mathrm{keV}, 0.129(.043) \mathrm{keV}$, and $0.18(.05) \mathrm{keV}$, as derived from tables given by Porquet et al. (2001). They all cover nicely the values obtained in the multi-T fit (Sect. 3.2).

\subsubsection{Density}

By definition (see Sect. 3.2), knowledge of the electron density $n_{\mathrm{e}}$ offers the possibility to derive the emitting volume, assuming a source with spatially constant $n_{\mathrm{e}}$.

The ratios (f/i) of C V, N VI, O VII, Ne IX, Mg XI, and Si XIII serve as density diagnostic. The line triplet of $\mathrm{SXV}$ is not 
resolved and given as one feature in Table 3. Their lines are located in the range from 5 to $45 \AA$ covered by LETGS. Here, I will focus on the ions N VI, O VII, and Ne IX. They are the stronger of the line triplets and well resolved.

Considering the measured line fluxes (see Table 3), the f/i ratios of N VI, O VII, and Ne IX are $\lesssim .46,0.15(.06)$ and $0.63(.07)$, respectively. These ratios result in densities of $\gtrsim 6.5 \times 10^{10} \mathrm{~cm}^{-3}$ for N VI, $n_{\mathrm{e}}=8.2(-2.4,+6.4) \times 10^{11} \mathrm{~cm}^{-3}$ for O VII, and of $n_{\mathrm{e}}=3.5(1.0) \times 10^{12} \mathrm{~cm}^{-3}$ for Ne IX (Porquet et al. 2001). This is in fair agreement with the values obtained from the multitemperature fit (see Table 2).

\section{Discussion}

In this section I will discuss my results and pay special attention to differences and agreements between my results and results given in former publications. It is difficult to conclude where differences come from: whether they originate in the use of three different instruments or different models, in erroneous interpretations, or in actual variation of the source.

The three obtained CIE-temperatures (0.127(.012), $0.264(.007)$, and $1.45(.43) \mathrm{keV}$ ) are in very good agreement with values established by Stelzer \& Schmitt (2004) $(0.172,0.272$, and $1.72 \mathrm{keV}$, respectively) and by Robrade \& Schmitt (2006) (0.24, 0.60, and $1.37 \mathrm{keV}$, respectively). To improve the shape op the continuum a blackbody component of $0.187(.016) \mathrm{keV}$ was introduced that replaces the temperature bin of $0.60 \mathrm{keV}$ found by Robrade \& Schmitt. From Table 2 in Günther et al. (2007) we notice that this CIE temperature component $(0.72 \mathrm{keV})$ is not very significant. The introduction of my blackbody component models the shape of the continuum from 5 to $15 \AA$ better. Thanks to that it offers the possibility to fit some weak line features of silicon and sulphur properly. It reduces $\chi^{2}$ significantly; from 537 (applying parameters given by Robrade \& Schmitt 2006) to 334 (this work) for 299 degrees of freedom and just covering the wavelength range from 5 to $35 \AA$ A. It is possible that my "blackbody" continuum describes a continuum formed by many weak lines not present in the databases.

My fitted value of $N_{\mathrm{H}}$ is higher than the values given by Stelzer \& Schmitt (2004) and Robrade \& Schmitt (2006). This higher value is necessary to suppress the appearance of lines above $40 \AA$ in the model that are absent in the data. A fit with a Gaussian at the theoretical position of the Si XII lines at $44 \AA$ (where no feature is visible) yields an upper limit of $7 \times 10^{-6}$ photons $\mathrm{cm}^{-2} \mathrm{~s}^{-1}$. These lines also appear in the model when using the parameter sets given by Stelzer \& Schmitt (2004) and Robrade \& Schmitt (2006), based on RGS, to describe the LETGS spectrum. Their parameters, however, were based on data sets limited to $35 \AA$, not covering the higher wavelength range where these lines are located.

The abundance pattern $(A / O)$ is quite well the same as for the two other papers, especially concerning the Model B in Stelzer $\&$ Schmitt (2004). Neon and nitrogen are enhanced. My carbon abundance relative to oxygen is a bit higher than theirs, but that is due to my higher $N_{\mathrm{H}}$ value. My absolute abundance values, however, are far higher (by a factor 2-3) than those given in the two other papers. This results in correspondingly lower values of the emission measures, as the quantity $A \times E M$ is robust. Despite the big difference in absolute values for the emission measure the overall emission measure shape agrees with Stelzer $\&$ Schmitt (2004) and the quantity $A \times E M$ is comparable for the three papers (Stelzer \& Schmitt 2004; Robrade \& Schmitt 2006; and this work).

The electron density $n_{\mathrm{e}}$, derived from O VII and Ne IX is high compared to electron densities observed in stellar coronae. My obtained $n_{\mathrm{e}}$ value of about $10^{12} \mathrm{~cm}^{-3}$ corresponds to values given in the other papers. It is related to the temperature regime around $.2 \mathrm{keV}$ and considered to be an indication for a process of accretion.

Although the overall pattern of the line fluxes and line flux ratios agree with Stelzer \& Schmitt (2004) and Kastner et al. (2002) it is wondering that even for strong features the absolute line flux values differ considerably. This is especially true for the line fluxes given by Stelzer \& Schmitt (2004) and Kastner et al. (2002). Thanks to the fact that the ratios agree better than the absolute values the conclusions drawn concerning the density, temperatures, and abundances are the same for the three papers. The underlying reason for the difference in line flux might be related to the use of different instruments or to the variability of the source, due to higher volumes of accreted material. The latter is suggested by the difference in line fluxes of $\mathrm{Ne} X$ and $\mathrm{Ne} I X$. The ratio $(\mathrm{f}+\mathrm{i}) / \mathrm{r}$ in He-like ions is close to 1 , indicating a plasma in collisional equilibrium.

The measured line positions are slightly shifted to higher wavelength. The corresponding velocity of about $30 \mathrm{~km} \mathrm{~s}^{-1}$ is higher than the satellite motion or $v_{\text {rad }}$ of TW Hya. The lines are broadened, in addition to the instrumental LSF, by an FWHM of $\sim 0.025 \AA$. This is broader than comparable lines in the coronal spectrum of Capella. Shift and broadening might be fingerprints of the velocities in the funneling accreted plasma from the disc to the stellar surface, although a calibration bias cannot be ruled out. In the latter case it might affect LETGS as well as RGS.

\section{Summary}

The overall conclusions based on the LETGS spectrum of TW Hya confirm the statements made by Robrade \& Schmitt (2006), Stelzer \& Schmitt (2004) and Kastner et al. (2002), concerning the dominance of a high density plasma $\left(n_{\mathrm{e}} \simeq 10^{12} \mathrm{~cm}^{-3}\right)$ at temperatures of about $0.2 \mathrm{keV}$. This is different from coronal cool star spectra for which higher temperatures and lower densities are determined (e.g., Raassen et al. 2003).

The abundances of neon $(10 \times)$ and nitrogen $(3 \times)$ are strongly enhanced in comparison with the oxygen abundance.

Thanks to the extended wavelength region of LETGS more information about the spectrum above $40 \AA$ was obtained, resulting in a somewhat higher value of the $N_{\mathrm{H}}$, i.e., the sum of the interstellar absorption and the circumstellar absorption just near TW Hya, compared to values used or obtained by Stelzer \& Schmitt (2004) and Robrade \& Schmitt (2006).

Differences in line fluxes between the papers Stelzer \& Schmitt (2004), Kastner et al. (2002), Ness \& Schmitt (2005) and this work are an indication for variability in mass accretion rate, but might also be due to instrumental differences.

An additional blackbody component is added to improve the multi-temperature fit and to describe a deficiency in continuum radiation between 5 and $15 \AA$ in the model.

Acknowledgements. The SRON National Institute for Space Research is supported financially by NWO.

\section{References}

Anders, E., \& Grevesse N. 1989, Geochim. Cosmochim. Acta, 53, 197 Arnaud, M., \& Rothenflug, R. 1985, A\&AS, 60, 425 
Feigelson, E. D., \& DeCampli, W. M. 1981, ApJ, 243, L89

Feigelson, E. D., Casanova, S., Montmerle, T., \& Guibert, J. 1993, ApJ, 416, 623

Gabriel, A. H., \& Jordan, C., 1969, MNRAS, 145, 241

Grevesse, N., \& Sauval, A.J. 1999, A\&A, 347, 348

Güdel, M., \& Telleschi, A. 2007, A\&A, 474, L25

Günther, H. M., \& Schmitt, J. H. M. M. 2008, A\&A, 481, 735

Günther, H. M., Schmitt, J. H. M. M., Robrade, J., \& Liefke, C. 2007, A\&A, 466, 1121

Herbst, W., \& Koret, D.L. 1988, AJ, 96, 1949

Huélamo, N., Figueira, P., Bonfils, X., et al. 2008, A\&A, 489, L9

Kaastra, J. S., Mewe, R., \& Nieuwenhuijzen H. 1996a, in UV and X-ray

Spectroscopy of Astrophysical and Laboratory Plasmas, ed. K. Yamashita, \& T. Watanabe (Universal: Academy Press, Inc., Tokyo), 411 (SPEX)

Kaastra, J. S., Mewe R., Liedahl, D. A., et al. 1996b, A\&A, 314, 547

Kastner, J. H., Huenemoerder, D. P., Schulz, N. S., et al. 2002, ApJ, 567, 434

Kelly, R. L. 1987, J. Phys. Chem. Rev. Data, 16
Mekkaden, M. V. 1998, A\&A, 340, 135

Mewe, R., Gronenschild, E. H. B. M., \& van den Oord, G. H. J. 1985, A\&AS, 62, 197

Mewe, R., Kaastra, J. S., \& Liedahl, D. A. 1995, Legacy, 6, 16 (MEKAL)

Ness, J.-U., \& Schmitt, J. H. M. M. 2005, A\&A, 444, L41

Perryman, M. A. C., Lindegren, L., Kovalevsky, J., et al. 1997a, A\&A, 323, L49

Perryman, M. A. C. 1997b, The Hipparcos and Tycho Catalogues, ESA SPseries, 1200

Porquet, D., Mewe, R., Dubau. J., et al. 2001, A\&A, 376, 1113

Raassen, A. J. J., Mewe R., Audard M., \& Güdel, M. 2003, A\&A, 411, 509

Reid, N. 2003, MNRAS, 342, 837

Robrade, J., \& Schmitt, J. H. M. M. 2006, A\&A, 449, 737

Rucinski, S. M., Matthews, J. M., Kuschnig, R., et al. 2008, MNRAS, 391, 1913

Setiawan, J., Henning, Th., Launhardt, R., et al. 2008, Nature, 451,38

Stelzer, B., \& Schmitt, J. H. M. M. 2004, A\&A, 418, 687

van Leeuwen, F. 2007, A\&A, 474, 653 